\title{
Three-dimensional effects of twinning in magnesium alloys
}

\author{
K. Hazeli ${ }^{1}$, J. Cuadra ${ }^{1}$, F. Streller ${ }^{4}$, C.M. Barr ${ }^{3}$, M.L. Taheri ${ }^{3}$, R. W. Carpick $^{4}$ and A. Kontsos ${ }^{1, *}$ \\ ${ }^{1}$ Department of Mechanical Engineering \& Mechanics, Drexel University, USA \\ ${ }^{2}$ Department of Materials Science \& Engineering, University of Pennsylvania, USA \\ ${ }^{3}$ Department of Materials Science \& Engineering, Drexel University, USA
}

${ }^{4}$ Department of Mechanical Engineering \& Applied Mechanics, University of Pennsylvania, USA

\begin{abstract}
This letter provides experimental evidence on the pronounced three-dimensional surface effects caused by tension twinning at early stages of plasticity of a magnesium alloy. To achieve this goal, multiscale mechanical testing was combined with strain field, texture and surface morphology quantification. The current investigation correlates crystallography-driven, grainscale surface roughening to the spatially heterogeneous micro- and macrostrain. It was found that tension twinning causes considerable surface extrusions and intrusions across multiple grains that are directly related to severe strain inhomogeneities.
\end{abstract}

Keywords: Twinning; Surface step; Strain localization; Digital image correlation (DIC); Local plasticity

*Corresponding author. E-mail: akontsos@coe.drexel.edu. 
Understanding twinning, including nucleation of different variants, their growth and interactions with other deformation modes, as well as detwinning, is a topic of increasing interest for hexagonal close-packed (hcp) alloys due to its role in plasticity, including anisotropy, hardening, damage incubation and failure [1]. Because of its relatively easy activation, much attention has been paid to $\{10 \overline{1} 2\}$ tension twinning [2-5]. Thompson and Millard [6] and Yang et al. [7] performed experimental analyses on cadmium and titanium respectively, based on which they suggested that lenticular twins resulted in step formations between two $\{10 \overline{1} 2\}$ lattice planes at the twin boundary. Furthermore, recent studies based on molecular dynamics simulations and electron backscattered diffraction (EBSD) data provided further evidence on twin nucleation [1,8-10]. Moreover, the statistical analysis of twin nucleation presented by Beyerlein et al. [1] indicated that grain boundary misorientations influence twin nucleation and growth. It has also been proposed that the stress states in the vicinity of grain boundaries dictate the prevalence of specific twin variants [11]. It has been further reported that the tendency of $\{10 \overline{1} 2\}$ tension twins to grow faster than contraction twins could be explained by the fact that the transverse propagation of $\{1101\}$ twins is hindered by the absence of low-energy mobile interfaces [5]. In addition to the considerable effect of twinning on ductility [12,13], a study by Kocks and Westlake [14] and another one by Hutchinson [15] suggested that the ductility of polycrystalline hcp metals is affected by local strain variations in neighboring grains. The basis of this relationship is the local strain inhomogeneities caused at boundaries of adjoined grains which, at high strain concentrations, may cause large tractions related to the onset of damage [16]. Such 
strain heterogeneities could be relieved by localized twinning and/or slip [17]. It has also been suggested that twinning is associated with crack formation at later stages of deformation [17-19]. Therefore, besides some of the known sources of crack initiation, such as surface roughness, inclusions, intermetallics and ductile/brittle phases in polycrystalline alloys [20], twinning, slip and strain inhomogeneities at the grain scale can be also envisioned as other sources of crack incubation and initiation [21]. In agreement with Muránsky et al. [22] and Barnett et al. [23], the current authors recently demonstrated [24] that the onset of the distinct plateau region in the stress-strain curve during compressive loading perpendicular to the $c$-axis is associated with profuse and spatially inhomogeneous twinning. In fact, this study showed the formation of distinct strain localization in the elastic-plastic transition. It has been also reported by the authors that, although tension twinning contributes to overall plasticity, it could further give rise to crack initiation towards the end of the fatigue life in Mg alloys [25].

Similar to the earlier study by Vaidya and Mahajan [26], in which evidence of slip on $\{11 \overline{2} 1\}$ planes prior to twinning was reported, Koike et al. [3] demonstrated that twinning occurs in grains with high basal dislocation activity. Therefore, as Hull [18] and more recently Ando et al. [17] suggested that localized strain inhomogeneities resulting from the activation of slip can presumably be relieved by twinning. It is also plausible that stress concentrations due to incompatibilities between twins and their surrounding matrix regions can be relaxed by slip $[17,26]$ or crack formation [12]. Despite the aforementioned progress in understanding twin formation and development, it is expected that the current knowledge could be greatly enhanced by a detailed characterization at the grain scale of the conditions that lead to twin formation [9]. 
In order to explain the relationship between grain-scale microstructural changes and deformation in alloys, a number of recent investigations relied on the use of the digital image correlation (DIC) method [27-31]. For instance, Carroll et al. [32] combined ex situ texture measurements with high-resolution strain field mapping obtained by DIC to explain strain localizations near grain boundaries. This study showed that some particular grains accumulate significant levels of plastic strain, while some of their immediate neighbors remained relatively undeformed. Furthermore, Dave et al. coupled incremental straining of nickel foils with optical microscopy and showed the significant effect of grain boundaries and orientation mismatches on localized strain distributions [30].

In this letter, full-field deformation measurements in Mg alloys were first performed at the grain scale. A cube-shaped specimen consisting of grains with most of their $c$-axis perpendicular to the loading direction was prepared from a commercial AZ31 plate. A region of interest (ROI) of 1 $\mathrm{mm}^{2}$ size was defined by using fiducial markers previously created by micro-hardness indentations. The sample was loaded utilizing an MTI/Fullam (SEMaster 1000 EBSD) stage at a compressive displacement rate of $0.5 \mathrm{~mm} \mathrm{~min}^{-1}$ up to about $2 \%$ overall strain. An optical microscope (Olympus $\mathrm{BH}-2)$ was fixed at $10 \times$ magnification to record image sequences during loading. The images were recorded by a 10 megapixel camera with a $25 \mathrm{~Hz}$ acquisition rate. The strain field evolution during loading was achieved by direct tracking of the microstructure using the DIC method [33]. The gray intensity (contrast) field captured by the camera was created by the alloy microstructure without any additional surface patterning. Two-dimensional deformation measurements were performed in a field of view of $900 \times 675 \mu \mathrm{m}^{2}$, which resulted in a maximum spatial resolution for deformation measurements of $11.25 \mu \mathrm{m}$ pixel $^{-1}$. The recorded digital images were post-processed using the commercially available ARAMIS GOM software (v6.30- 
4). A smaller ROI of $196 \times 239 \mu \mathrm{m}^{2}$ at a specific location was analyzed to compute more accurate full-field and point strains. The surface morphology in the ROI was inspected postmortem by non-contact white light interferometry using a Zygo surface profilometer (model 6300 ) at $20 \times$ magnification. As a result of using the Mirau objective lens, a lateral resolution of $750 \mathrm{~nm}$ and a vertical resolution of $0.1 \mathrm{~nm}$ were achieved. Note that, in order to prevent changes on the deformed specimen surface caused by chemical agents, the orientation and topography maps of the marked regions for the deformed samples were performed without any further polishing or etching.

To validate the surface morphology observations at the grain scale, compression tests on cylindrical specimens of (ASTM) standard dimensions were also performed. In this case, samples were flattened on one side to allow post-mortem microstructure analyses and then mechanically compressed at room temperature with a strain rate of $4.5 \times 10^{-4} \mathrm{~s}^{-1}$. Prior to testing, the specimens were subjected to the polishing procedure described previously [34]. Fiducial markers on the polished surface were produced by microhardness indentation. The surface morphology in the ROI $\left(250 \times 250 \mu m^{2}\right)$ was quantified using the interferometry method used for the grain-scale tests. Full-field strain fields were measured using DIC on the cylindrical surface of the tested specimens. The full-field strain measurement setup was capable of resolving strains with an accuracy of $\pm 150 \mu \mathrm{m} \mathrm{m}^{-1}$ and a resolution of $20 \mu \mathrm{m} \mathrm{pixel}{ }^{-1}$.

Figure 1(a) shows twin nucleation and growth observed in situ by the optical microscope on the grain-scale specimen subjected to compression by the miniature loading stage. Figure 1(b) displays the corresponding full-field strain evolution parallel to the loading direction, marked as global strain. Figure 1(c) plots the average global strain as a function of time. It also presents 
point-to-point measured strain values across the twin region highlighted in Figure 1a and b. This measurement shows how the local strain field within a grain develops as a result of twinning. The measured local and global strains demonstrate that, while the sample undergoes compression (see the black line in Fig. 1(c)), favorable grains (G1 and G2) for twinning, according at least to the reported high Schmid factor (SF) criterion [35], display large tensile strains exactly at twin locations. Relevant recent investigations by the authors on the onset of plastic flow reported that twinning indeed starts at very early stages of deformation for highly textured samples [24]. It has also been proposed by the current authors [34] that strain localizations associated with twinning first initiate at grains with grain orientation closer to that of the basal plane i.e. at grains with larger SF values [36,37]. It is of great significance to note here that the presented systematic characterization method enabled the direct measurement of grain-scale strain fields associated with twinning in Mg alloys.

Although the theoretical maximum tensile strain caused by twinning in Mg single crystal has been reported to be $6.5 \%$ [38], the current results provide locally and on average considerably larger values which are suspected to be related to 3-D surface effects of twinning. It is also important to note that the local strains measured in the transverse direction with respect to the long axis of the observed twins are tensile and not related to the shear component (which can in general be quantified in-plane by the DIC algorithm), therefore implying that comparisons with theoretical values are not feasible at least for the measurements reported herein. It is known in the DIC-related literature that out-of-plane motion directly affects any 2-D (in-plane) strain measurements. This was verified in Figure 1(e), which shows examples of pronounced surface morphology changes (differences in height are represented by a color bar in micrometers) obtained using the interferometry method. Large extrusions and intrusions in grain regions 
dominated by twinning revealed a complex surface relief. The formation of such surface steps due to twinning were previously reported to occur in later stages of plasticity during compression of Mg alloy samples [17]. However, to the best of our knowledge, this is the first study that reports surface step formation as a result of twinning and its associated strain fields at early stages of plasticity in Mg alloys. Similar to the results reported in this letter, out-of-plane components of strain due to slip and twinning were observed during four-point bending experiments on a titanium alloy [7].

Figure 2(a) demonstrates the yielding and post-yielding mechanical behavior in the stress-strain curve obtained by compression of cylindrical specimens. In order to investigate the microscale plasticity at the onset of yielding, the applied loading was interrupted while the strain was less than $1 \%$. Figure 2(b) presents the average full-field strain evolution along the loading direction for the strain stages marked with numbers in Figure 2(a). The strain maps suggest that the onset of yielding is coincident with distinct strain localizations. A more detailed description of such strain localizations is given by the authors in Ref. [24]. The results in Figure 2c and d validate the findings reported in Figure 1 that relate early plastic deformation to pronounced surface steps at the grain scale. Thorough microstructural examination of regions from inside the strain band shown in Figure 2(b) revealed surface steps quantified by the height vs. distance curve (along the points numbered as i and ii in Fig. 2(c)), which provides the size of extrusions and intrusions on the surface of the one-side-flattened cylindrical specimen. Moreover, Figure 2e-g provide the texture, SF values and kernel average misorientation (KAM) map, respectively, for the same ROI. It can be seen in Figure $2 \mathrm{f}$ that the grain with an SF of 0.25 did not undergo twinning, unlike the one with an SF of 0.47 , which contains several twins. This observation provides some evidence of the role of SF in twinning [1]. Additionally, Figure 2g shows distinct areas with 
pronounced local misorientation marked with white dashed ellipses near the grain that twinned. Such KAM maps are constructed by measuring the degree of misorientation between a measured point and all its surrounding neighbors, and have been related to the stored strain energy $[35,39,40]$. It is evident via the KAM that the density of dislocations at critical intersections of grain boundaries and twins (marked with solid black arrows in Fig. $2 \mathrm{~g}$ ) is relatively higher. This observation may support the probabilistic twin nucleation model for hcp polycrystalline metals proposed by Beyerlein and Tomé [9], while it also agrees with the model suggested by Meyer et al. [41] and the experimental study by Koike et al. [3], which suggest that stress concentrations are caused by the pronounced activation of basal slip prior to twinning. In agreement with a recent study by Balogh et al. [42], where stress gradients in the vicinity of twin boundaries were reported, the reported experimental results in this letter suggest that twin boundaries can have variable states of stress (strain). For example, Figure 2(d) shows the occurrence of several twins in one grain and demonstrates the significant variance in the size of such twin-related surface steps, providing a strong indication that twin dislocations could also be driven by variable stresses [42].

The twin path in relation to local misorientation, SF values and surface step geometry is investigated in Figure 3. It is shown in this figure that twins tend to form in grains with high SF values (0.46-0.48), while thickening occurs in energetically favorable paths (longer side of the grain). Moreover, it is expected that, as local internal stresses increase due to dislocation pile ups and activation of non-basal slip, subgrain recrystallization nuclei are formed as a result of $\phi$ and $\alpha+a$ dislocation interactions [43]. Figure 3(c) depicts the morphological effects related to surface step formation due to twinning on the examined ROI, which shows a tangent as high as $87.04^{\circ}$, with a height of $379.05 \mathrm{~nm}$. 
The grain-scale results presented in this letter combined in situ observations of twin nucleation and associated strain and morphological measurements, which suggest that twin formation is directly related to strain inhomogeneities among grains in Mg alloys. A closer inspection of the results in Figures 1-3 reveals significant strain and surface gradients within grains and near grain boundaries which can be conceivably reduced by secondary twins [17]. This letter further demonstrates the relationship between surface morphology evolution during compression of $\mathrm{Mg}$ alloys and twinning. Both in situ and ex situ grain-scale observations were used to demonstrate that twinning is responsible for large intrusions and extrusions that form inside and around twinfavorable grains. Direct evidence and quantification were provided to illustrate the 3-D nature of such twin-induced effects, which are very likely to be associated with both macroscale and microscale plasticity and fracture effects.

A.K. would like to acknowledge financial support from NSF CMMI Award No. 1434506.

M.L.T. gratefully acknowledges funding from the Department of Energy through contract \# NE0000315, and from the US Nuclear Regulatory Commission through contract \# NRC-38-10928.

\section{References}

[1] I. Beyerlein, L. Capolungo, P. Marshall, R. McCabe, C. Tomé, Philosophical Magazine, 90 (2010) 2161-2190.

[2] A. Serra, D.J. Bacon, Philosophical Magazine A, 73 (1996) 333-343.

[3] J. Koike, Y. Sato, D. Ando, Materials transactions, 49 (2008) 2792. 
[4] H. El Kadiri, J. Baird, J. Kapil, A. Oppedal, M. Cherkaoui, S.C. Vogel, International Journal of Plasticity, 44 (2013) 111-120.

[5] L. Leclercq, L. Capolungo, D. Rodney, Materials Research Letters, (2014) 1-8.

[6] N. Thompson, D.J. Millard, Philosophical Magazine Series 7, 43 (1952) 422-440.

[7] Y. Yang, L. Wang, T.R. Bieler, P. Eisenlohr, M.A. Crimp, Metall and Mat Trans A, 42 (2011) 636-644.

[8] C.D. Barrett, H. El Kadiri, Acta Materialia, 63 (2014) 1-15.

[9] I. Beyerlein, C. Tomé, Proceedings of the Royal Society A: Mathematical, Physical and Engineering Science, 466 (2010) 2517-2544.

[10] J. Wang, I. Beyerlein, C. Tomé, International Journal of Plasticity, 56 (2014) 156-172.

[11] I. Beyerlein, J. Wang, M. Barnett, C. Tomé, Proceedings of the Royal Society A:

Mathematical, Physical and Engineering Science, 468 (2012) 1496-1520.

[12] M. Yoo, Metallurgical Transactions A, 12 (1981) 409-418.

[13] M. Barnett, Materials Science and Engineering: A, 464 (2007) 1-7.

[14] U. Kocks, D. Westlake, AIME MET SOC TRANS, 239 (1967) 1107-1109.

[15] J. Hutchinson, Metallurgical Transactions A, 8 (1977) 1465-1469.

[16] T. Bieler, P. Eisenlohr, F. Roters, D. Kumar, D. Mason, M. Crimp, D. Raabe, International Journal of Plasticity, 25 (2009) 1655-1683.

[17] D. Ando, J. Koike, Y. Sutou, Acta Materialia, 58 (2010) 4316-4324.

[18] D. Hull, Acta Metallurgica, 8 (1960) 11-18.

[19] F. Yang, S. Yin, S. Li, Z. Zhang, Materials Science and Engineering: A, 491 (2008) 131-136.

[20] S. Suresh, Fatigue of Materials. Cambridge: Cambridge University Press; 1998. 
[21] R. Zeng, E. Han, W. Ke, W. Dietzel, K.U. Kainer, A. Atrens, International journal of fatigue, 32 (2010) 411-419.

[22] O. Muránsky, M. Barnett, D. Carr, S. Vogel, E. Oliver, Acta Materialia, 58 (2010) 1503-1517.

[23] M.R. Barnett, M.D. Nave, A. Ghaderi, Acta materialia, 60 (2012) 1433-1443.

[24] K. Hazeli, J. Cuadra, P.A. Vanniamparambil, A. Kontsos, Scripta Materialia, 68 (2013) 83-86.

[25] K. Hazeli, H. Askari, J. Cuadra, F. Streller, R.W. Carpick, H.M. Zbib, A. Kontsos, International Journal of Plasticity.

[26] S. Vaidya, S. Mahajan, Acta Metallurgica, 28 (1980) 1123-1131.

[27] D. Raabe, M. Sachtleber, Z. Zhao, F. Roters, S. Zaefferer, Acta Materialia, 49 (2001) 34333441.

[28] D. Raabe, M. Sachtleber, H. Weiland, G. Scheele, Z. Zhao, Acta Materialia, 51 (2003) 15391560.

[29] Z. Zhao, M. Ramesh, D. Raabe, A. Cuitino, R. Radovitzky, International Journal of Plasticity, 24 (2008) 2278-2297.

[30] S. Dave, X. Song, F. Hofmann, K. Dragnevski, A. Korsunsky, Procedia Engineering, 1 (2009) 197-200.

[31] A. Tatschl, O. Kolednik, Materials Science and Engineering: A, 339 (2003) 265-280.

[32] J.D. Carroll, W. Abuzaid, J. Lambros, H. Sehitoglu, International journal of fatigue.

[33] M.A. Sutton, J.-J. Orteu, H.W. Schreier, IMAGE CORRELATION for Shape, Motion and Deformation Measurements: Basic Concepts, Theory and Applications. Berlin: Springer; 2009. [34] K. Hazeli, A. Sadeghi, M.O. Pekguleryuz, A. Kontsos, Materials Science and Engineering: A, 578 (2013) 383-393. 
[35] Y. Takayama, J.A. Szpunar, Materials transactions, 45 (2004) 2316-2325.

[36] S.H. Park, S.-G. Hong, J.H. Lee, C.S. Lee, Materials Science and Engineering: A, 532 (2012) 401-406.

[37] X. Lou, M. Li, R. Boger, S. Agnew, R. Wagoner, International Journal of Plasticity, 23 (2007) 44-86.

[38] S. Kleiner, P. Uggowitzer, Materials Science and Engineering: A, 379 (2004) 258-263.

[39] S. Yi, H.-G. Brokmeier, D. Letzig, Journal of alloys and compounds, 506 (2010) 364-371.

[40] K. Hazeli, A. Sadeghi, M. Pekguleryuz, A. Kontsos, Materials Science and Engineering: A, 589 (2013) 275-279.

[41] M. Meyers, O. Vöhringer, V. Lubarda, Acta materialia, 49 (2001) 4025-4039.

[42] L. Balogh, S. Niezgoda, A. Kanjarla, D. Brown, B. Clausen, W. Liu, C. Tomé, Acta Materialia, (2013).

[43] D. Yin, K. Zhang, G. Wang, W. Han, Materials Science and Engineering: A, 392 (2005) 320325.

Figure 1. (a) Optical microscope images of twin nucleation and growth obtained in situ. (b) Fullfield strain maps obtained by DIC with overlaid grain structure. (c) Local (for two different grains) and global (average) strain evolution in time. (d) Measured SF values in the ROI. (e) 3-D views of the surface contour displaying grain-scale extrusions and intrusions, and their close relationship to twinning. 
Figure 2. (a) Interrupted stress-strain curve ( $\varepsilon<1 \%$ of total strain) for a cylindrical compression sample. (b) Full-field longitudinal (parallel to the loading axis) strains for seven increments marked on the stress-stain curve. (c) Surface step profile from a line scan identified by points i and ii in the morphology map in (b) obtained from white-light interferometry. (e) IPF for the same set of grains. (f) Corresponding SF values and (g) KAM maps.

Figure 3. (a) KAM before deformation, (b) SF contour after deformation and (c) 3-D effects of twinning are evident on the surface and its corresponding edges in the ROI. 
a
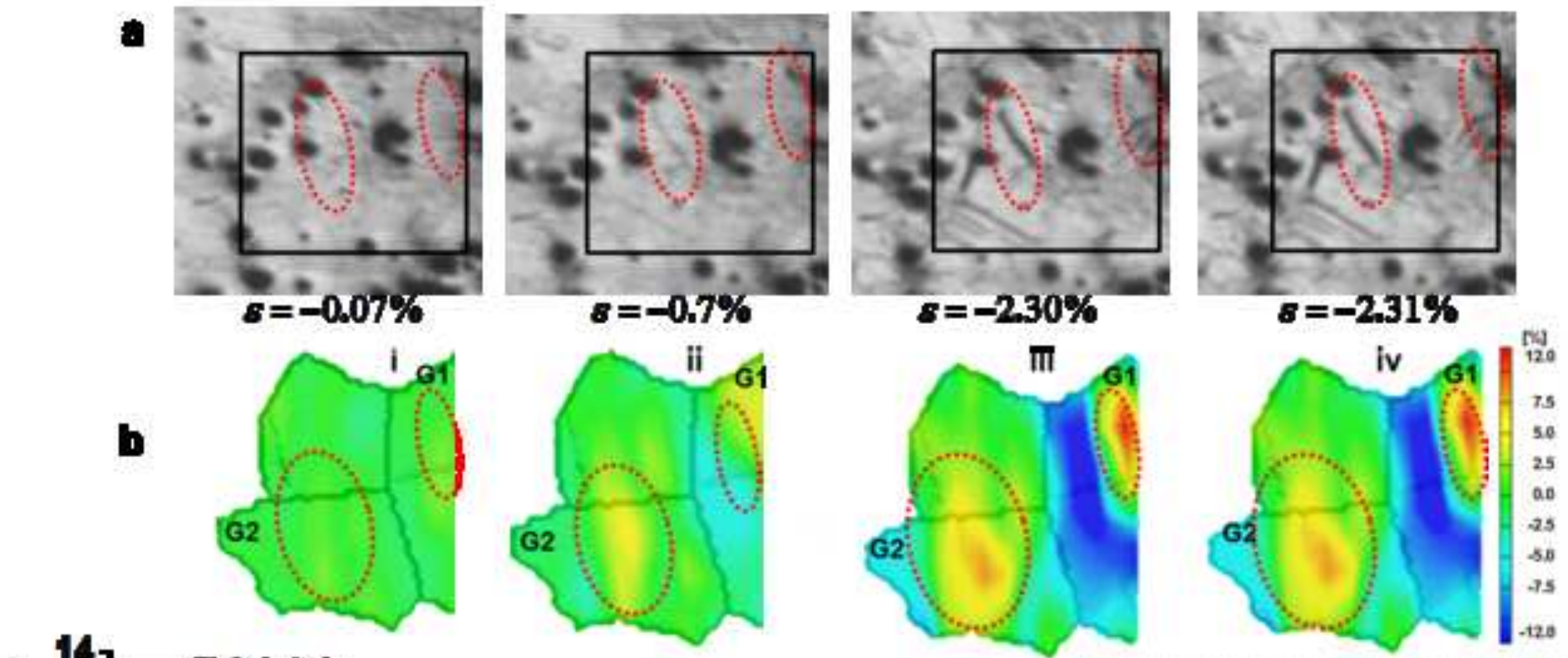
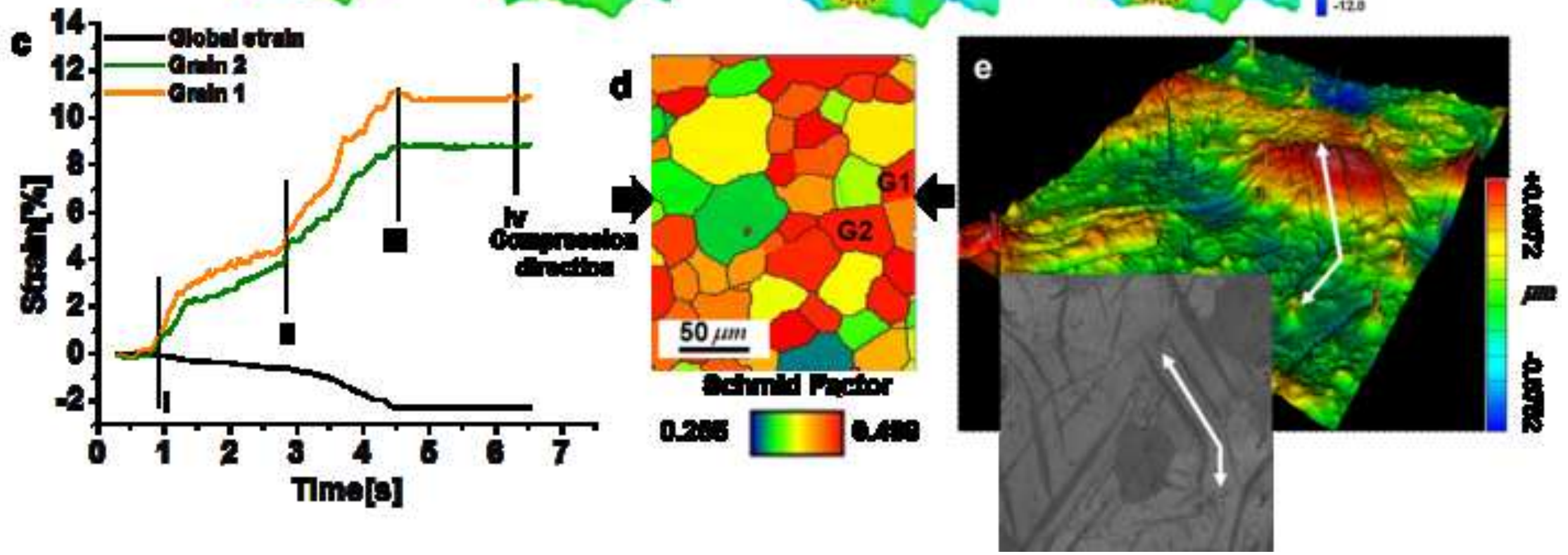
Fig. 2
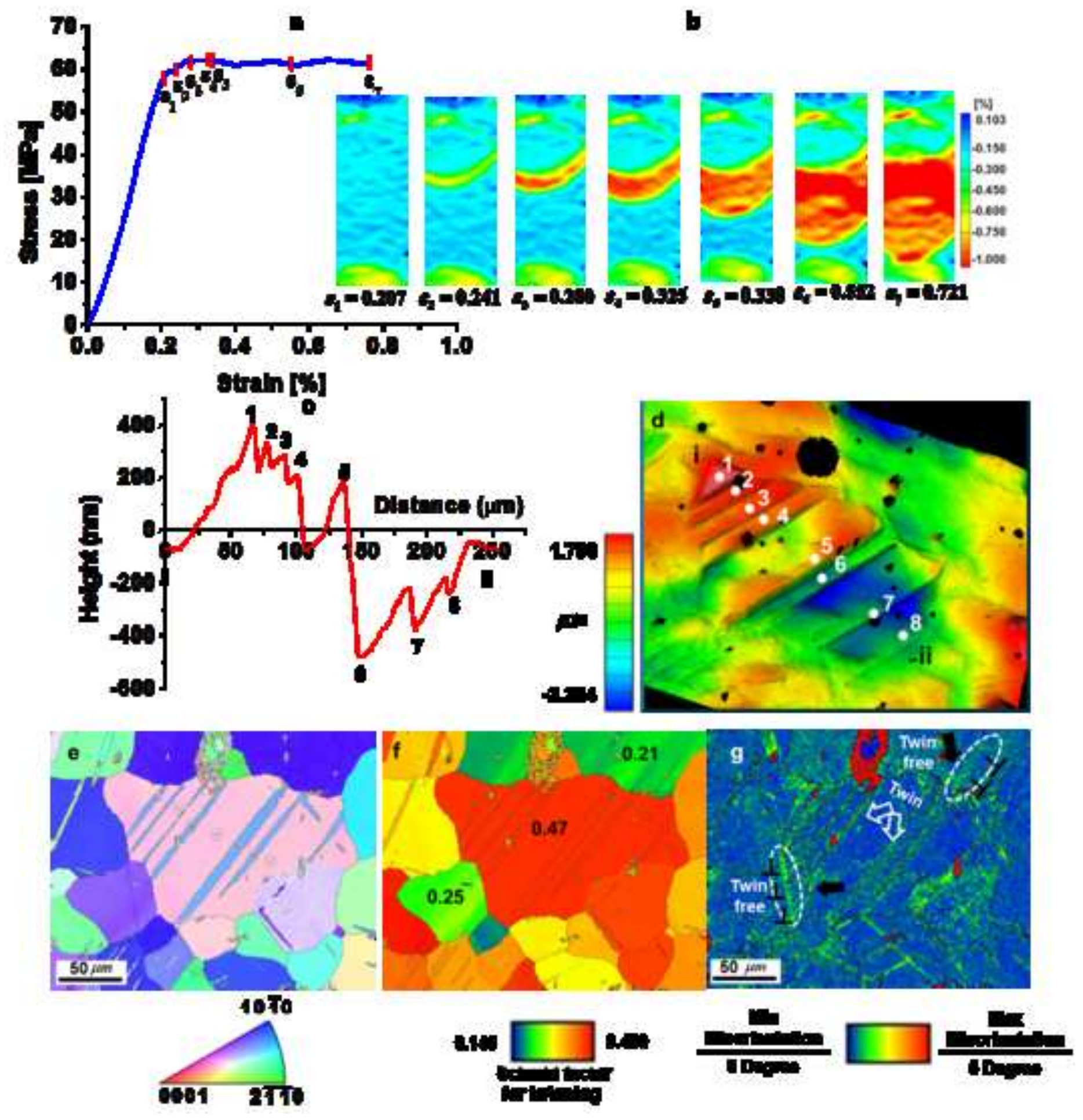


\section{Betare Defantion}

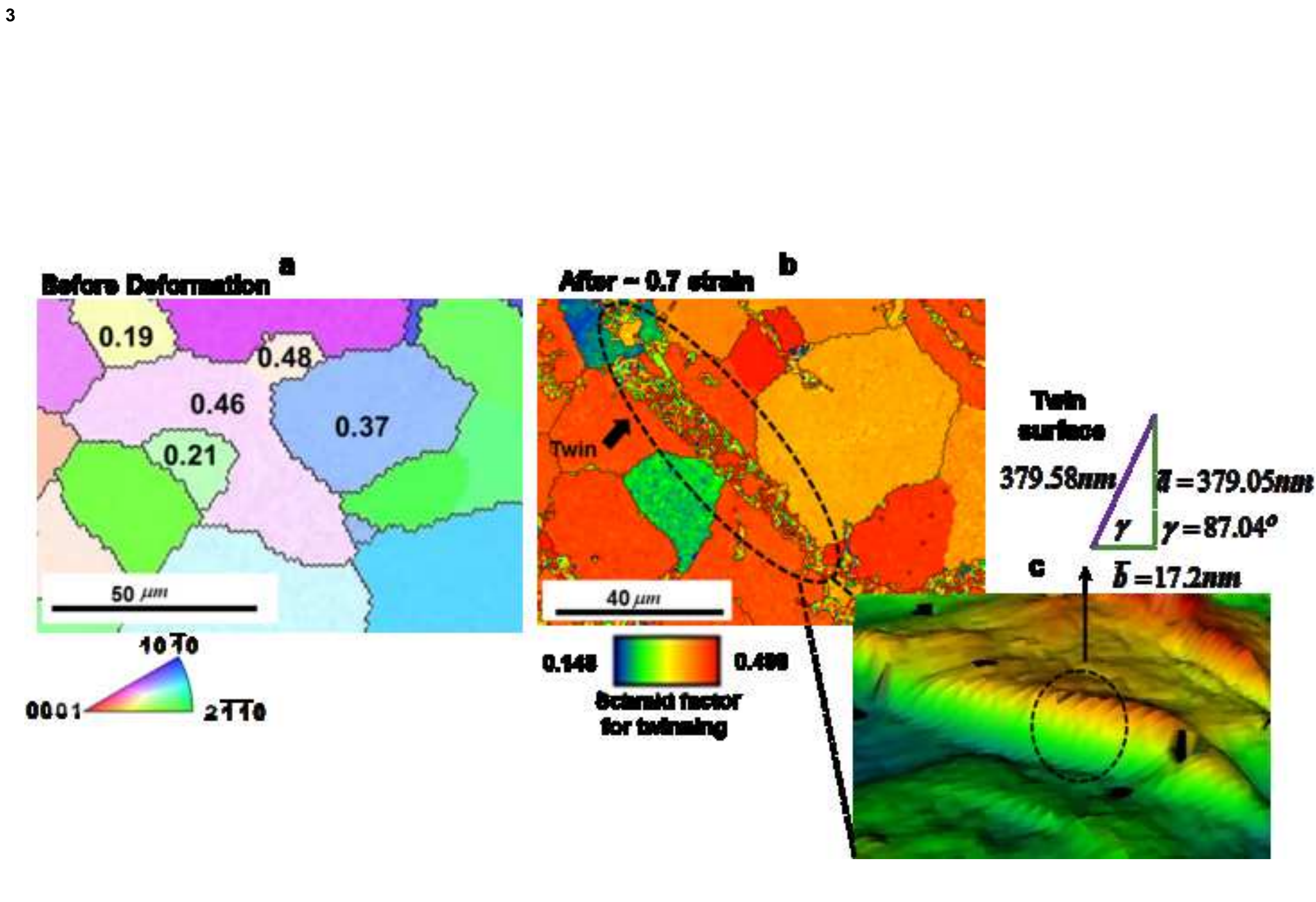

Mter - 0.7 h b
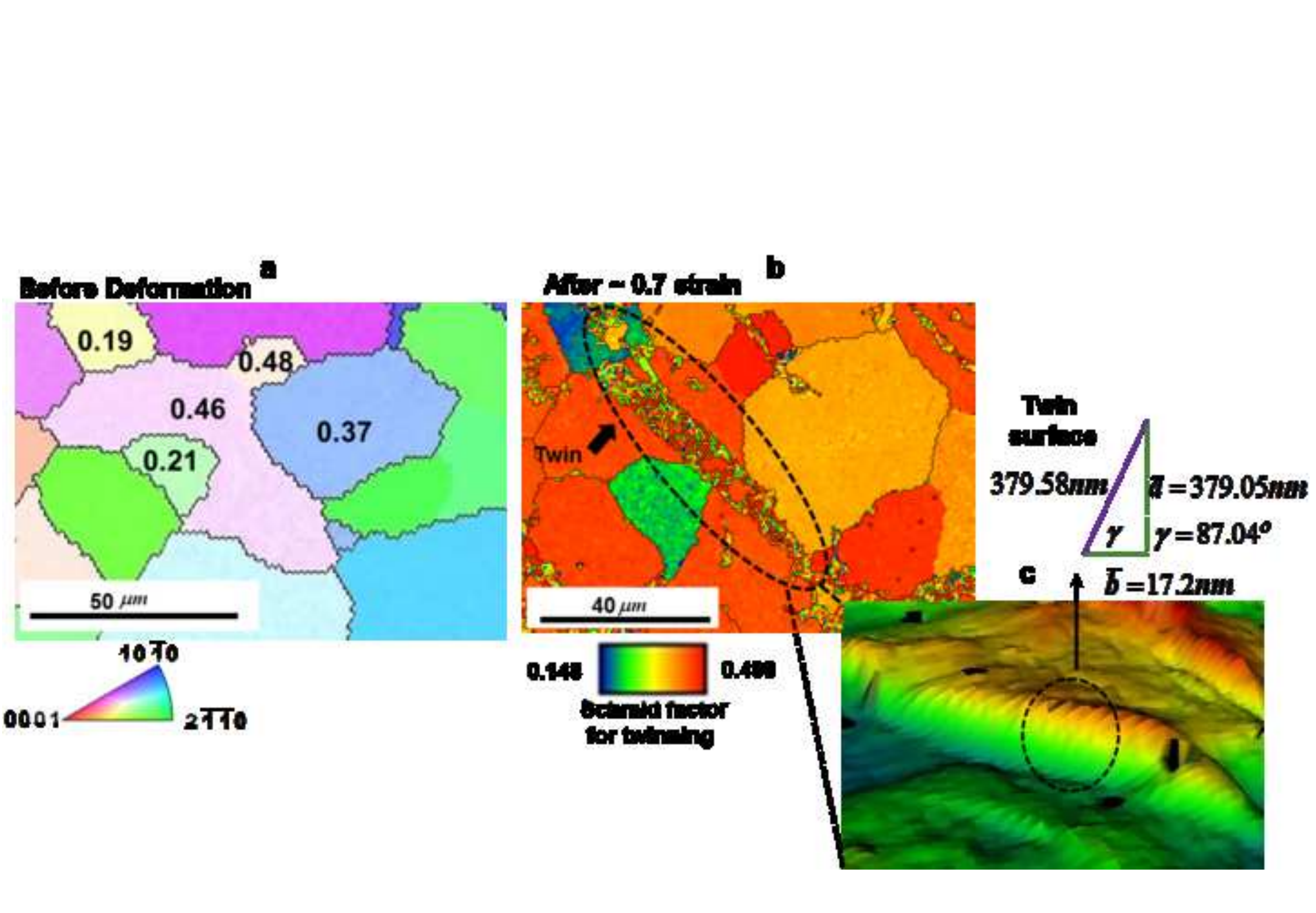

.

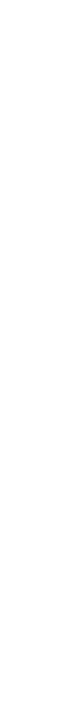

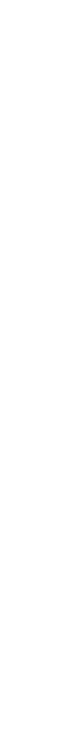

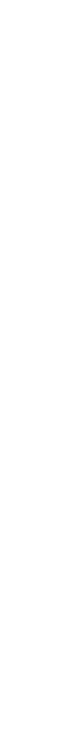

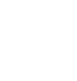

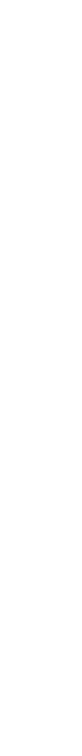

(1)

(1)

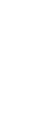

